\title{
Looking back at the past and projecting ahead to the future
}

\author{
Hikmet Budak ${ }^{1}$
}

Published online: 1 August 2015

(C) Springer-Verlag Berlin Heidelberg 2015

I am greatly honored to become the new Editor in chief of Functional \& Integrative Genomics, taking over this esteemed position from Prof. Rudi Appels, who has served remarkably and with humble dedication as the Editor in chief of this journal for many years. Filling his shoes will not be an easy task, but I am confident that with valuable contributions of the entire scientific community and distinguished Editorial Board members, we will succeed in taking this journal from its current status to new heights.

Since its establishment in May 2000, Functional \& Integrative Genomics has contributed vastly to the genomic studies of organisms. Many small genomes have been published; the transcriptomic, proteomic, and metagenomic aspects of various organisms have been addressed. Since then, the scope of Functional \& Integrative Genomics as a research area has evolved and expanded very rapidly into a highly specialized field. Now, let us return to the present and even project ahead to the future. In this first editorial, I would like to share with the readers and authors some of new directions of this journal. Presently, this journal is very committed to a wide range of studies concerning genomes and genomic functions, encompassing the biological processes involved. To begin with, I would like to expand the academic disciplines that this journal is currently focused on to include new functional genomics tools, genome analysis, and systems biology, areas in which research has increased in leaps and bounds over the last few years. We welcome genomic studies concerning all kingdoms of life, and the journal will now focus on parallels drawn in the genomic, proteomic, and metagenomic processes. This will enable rapid discovery and validation of novel genomic functions within various organisms and species. Organellar genomics from different organisms will be especially emphasized given its importance following its recent emergence as a hot topic of research, and potential special issues will focus solely on this topic.We will also welcome highly comprehensive review articles and letters combining the current findings with an integrative perspective to stimulate new scientific research avenues.

In line with the current research, the scope of the journal will inevitably be slightly shifted to genomics data sharing and web tools for all organisms, to provide more exceptional papers for our audience, thus, improving the journal content. I am well aware of the commitment I have undertaken as the Editor in chief of a journal focusing on the most recent advances in genomics research. Along with my Editorial Board members and reviewers, I will do my utmost to fulfill this commitment. I would like to take this opportunity to thank the entire genomics community for their relentless efforts and collaboration to produce the scientific articles and the Editorial Board for welcoming me in my new role.

I look forward to improving the quality of the journal, increasing its output, defining its course in research topics, and collaborating with the Editorial Board to turning Functional \& Integrative Genomics into a high impact-factor journal with outstanding research content.

Hikmet Budak

August 2015
Hikmet Budak

budak@sabanciuniv.edu

1 Molecular Biology, Genetics and Bioengineering, Sabanci University, University Cad. No:27, Tuzla, 34956 Istanbul, Turkey 